\title{
Hausärztliche Versorgung Patienten mit abdominaler Adipositas haben ein hohes Risiko
}

Von J. SChOlZE, W. Lilienthal, P. BRAMLAGE

\author{
Ein erhöhter Bauchumfang ist ein \\ Maß für die abdominale Adipositas. \\ In einer epidemiologischen Erhebung \\ in der hausärztlichen Praxis wur- \\ de gezeigt, dass bei Patienten mit \\ abdominaler Adipositas eine Reihe \\ kardiovaskulärer Risikofaktoren und \\ Endorganschäden vorliegt.
}

— Der Body-Mass-Index (BMI) wird im Allgemeinen als Ausdruck eines erhöhten Körperfettanteils angesehen, der Bauchumfang dagegen als Maß für die Verteilung des Körperfetts. In Deutschland sind etwa $30 \%$ aller Patienten übergewichtig (BMI $\geq 25 \mathrm{~kg} / \mathrm{m}^{2}$ ) und weitere $20 \%$ adipös (BMI $\geq 30 \mathrm{~kg} / \mathrm{m}^{2}$ ), $50 \%$ haben einen erhöhten Bauchumfang (Männer $\geq 102 \mathrm{~cm}$, Frauen $\geq 88$ $\mathrm{cm})$. Die abdominale Adipositas ist damit einer der häufigsten kardiometabolischen Risikofaktoren in der niedergelassenen Praxis.

Häufig geht die abdominale Adipositas mit weiteren Erkrankungen wie arterielle Hypertonie, Diabetes mellitus Typ 2 und Dyslipidämie einher. Die Therapie dieser Erkrankungen sowie die normnahe Einstellung von Blutzucker und Lipiden ist in der täglichen Praxis eine Herausforderung und oft versagen gängige Therapiestrategien.

Ziel dieser Erhebung war es daher, die Häufigkeit von begleitenden kardiometabolischen Risikofaktoren in der niedergelassenen Praxis zu dokumentieren, die Schnittmenge aller drei Risikofaktoren zu quantifizieren und die Versorgungssituation der Patienten zu untersuchen.

\section{Ergebnisse}

Die Daten von insgesamt 49433 Patienten mit abdominaler Adipositas gelangten zur Auswertung. 70,9\% $(\mathrm{n}=$

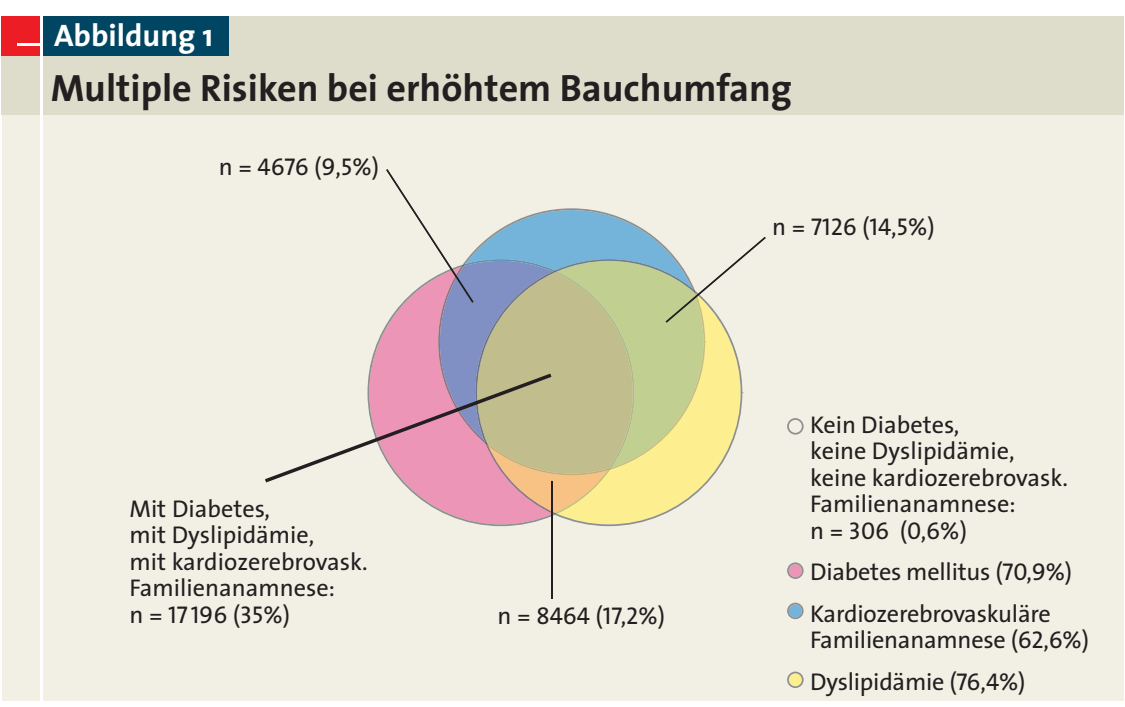

Abb. 1 Abdominale Adipositas tritt häufig gemeinsam mit Typ-2-Diabetes, Dyslipidämie oder Hypertonie auf (Gesamtstichprobe: $n=49433$ ).

35 072) dieser Patienten hatten nach Arztangaben gleichzeitig einen Diabetes mellitus Typ 2, 76,4\% eine Dyslipidämie (niedriges HDL-C oder erhöhte Triglyzeride). Nur bei $0,6 \%$ aller Patienten mit abdominaler Adipositas lagen weder Diabetes noch Dyslipidämie oder kardiovaskuläre Ereignisse in der Familienanamnese vor. 35,0\% aller Patienten $(\mathrm{n}=17196)$ wiesen dagegen eine Dreifachkombination aus den genannten Risikofaktoren auf (Abb. 1)

Eine Multimedikation mit Antihypertensiva, Antidiabetika, Lipidsenkern und Thrombozytenaggregationshemmern war die Regel. Häufig konnten aber die Zielwerte für z.B. den Blutzucker $\left(\mathrm{HbA}_{1 \mathrm{c}}<6,5\right.$ oder $\left.<7,0 \%\right)$ nicht erreicht werden.

\section{Fazit}

Abdominale Adipositas (gemessen am Bauchumfang) ist ein wesentlicher Risikofaktor für kardiovaskuläre Erkran- kungen. Sie tritt häufig gemeinsam mit Diabetes mellitus Typ 2, (atherogener) Dyslipidämie und arterieller Hypertonie auf. Mit den verfügbaren Therapiestrategien gelingt es nicht immer, die empfohlenen metabolischen Zielwerte zu erreichen.

\section{Literatur siehe Langfassung}

\section{Für die Verfasser:}

Prof. Dr. med. Jürgen Scholze

Charité - Universitätsmedizin Berlin Medizinische Poliklinik

Charitéplatz 1

D-10117 Berlin

E-Mail:juergen.scholze@charite.de

\section{- Die Erhebung wurde unterstützt von der} Sanofi-Aventis Deutschland $\mathrm{GmbH}$, Berlin.

Die ausführliche Langfassung der Erhebung erscheint im vierteljährlich publizierten Originalienergänzungsband der MMW, der im Rahmen des Abonnements der Zeitschrift bezogen werden kann. Die Langfassung ist erhältlich gegen 10,- Euro als Verrechnungsscheck auf Anfrage oder online unter http://www.mmw.de 